\title{
Assessment of Plain and Hyperbaric Solutions of Ropivacaine in Patients Receiving Spinal Anaesthesia for Elective Surgeries: A Comparative Study
}

\author{
Ajay Batra \\ Associate Professor, Department of General Anesthesia, \\ Narayan Medical College and Hospital, Jamuhar, Rohtas, Sasaram, Bihar, India.
}

\begin{abstract}
Background: Very little research is done in case of ropivacaine, $\mathrm{n}$ terms of its intrathecal use. Sensory block of very variable amount and quality has been produced by varying concentration of ropivacaine and ropivacaine with and without glucose as shown by past studies in the literature. Hence; we comparatively evaluated the effectiveness of plain and hyperbaric ropivacaine for providing subarachnoid block in patients undergoing elective surgeries.

Materials \& Methods: The present study was carried out in the department of general surgery and included 80 patients undergoing elective surgical procedures under spinal anaesthesia. All the patients were randomly divided into two study groups. First group included patients who received plain ropivacaine solution while the other group included patients who received ropivacaine admixed with glucose. Aseptic preparation of the solutions of anaesthesia was done before administration of the block. Bromage scale was used for defining the criteria of the motor nerve block. Recording of the pulse rate was done at regular time intervals following injection of the block. Complete post-operative follow-up of all the patients was done at regular time intervals all the data records were collected, summarized and analyzed.

Results: Mean age of the patients in group receiving plain and hyperbaric anaesthetic solution was 61 and 59 years respectively. Mena height of the patients in the two study groups was 166.5 and $164.2 \mathrm{~cm}$ respectively. 72 and $70.5 \mathrm{~kg}$ was the mean weight of the patients in the two study groups.
\end{abstract}

\section{INTRODUCTION}

In terms of its intrathecal use, very little research is done in case of ropivacaine. Sensory block of very variable amount and quality has been produced by varying concentration of ropivacaine and ropivacaine with and without glucose as shown by past studies in the literature. The main possible reason for this may be due to inadequate distribution of block in that patients. ${ }^{1,2}$ Studies conducted in the near past showed that satisfactory results in terms of producing satisfactory level of analgesia are be obtained by plain ropivacaine but element of doubt exists in terms of other agents when used in plain concentrations. ${ }^{3,4}$ Hence; we comparatively evaluated the effectiveness of plain and hyperbaric ropivacaine for providing subarachnoid block in patients undergoing elective surgeries.
Non-significant results were obtained while comparing the demographic parameters in between the two study groups. Mean duration of onset of anaesthesia to T10 was 10.5 minutes and 5.2 minutes and patients in plain and hyperbaric group respectively. Significant differences were obtained while comparing the mean time for motor regression and mean time for sensory regression in between the two study groups.

Conclusion: In comparison with the hyperbaric type, plain ropivacaine has been observed to be associated with less favourable pattern of block.

Key Words: Hyperbaric, Plain, Rupivacaine.

\section{${ }^{*}$ Correspondence to:}

\section{Dr Ajay Batra}

Associate Professor, Department of General Anesthesia,

Narayan Medical College and Hospital,

Jamuhar, Rohtas, Sasaram, Bihar, India.

Article History:

Received: 21-07-2016, Revised: 06-08-2016, Accepted: 10-08-2016

\begin{tabular}{|l|c|}
\hline \multicolumn{2}{|c|}{ Access this article online } \\
\hline Website: & Quick Response code \\
www.ijmrp.com & \\
\hline DOI: & \\
10.21276/ijmrp.2016.2.5.016 & \\
\hline
\end{tabular}

\section{MATERIALS \& METHODS}

The present study was carried out in the department of general surgery and included 80 patients undergoing elective surgical procedures under spinal anaesthesia and came under the category of ASA grade I-II. Ethical approval was taken from the institutional ethical committee and written consent was obtained in written from all the patients after explaining them in details the entire research protocol. Pre-medications was given to the patients which included oral temazepam and other few drugs. Regular pre-operative monitoring of the clinical parameters of the patients was done by pulse oximetry, ECG, blood pressure. Access was gained to eh venous route of the patients for mentoring the invasive parameters. 25-swg needle was used for 
making the lumbar puncture using a midline approach. All the patients were randomly divided into two study groups. First group included patients who received plain ropivacaine solution while the other group included patients who received ropivacaine admixed with glucose. Aseptic preparation of the solutions of anaesthesia was done before administration of the block. Proper mixing of the ropivacaine solution was done with the glucose in patients receiving glucose admixed ropivacaine solution. Supine position of the patients was achieved after giving them adequate instructions in the immediate time after achieving and injecting the anaesthetic solution and this time was defined as Zero time as all the parameters alterations and sensory and motor values were taken from this point. After this time, readings of all the data of the clinical and haematological parameters were done. Bromage scale was used for defining the criteria of the motor nerve block. Recording of the pulse rate was done at regular time intervals following injection of the block.

Following the regression of the sensory nerve block, mobilization of the patients was encouraged under proper supervision. Complete post-operative follow-up of all the patients was done at regular time intervals all the data records were collected, summarized and analyzed. All the results were assessed by SPSS software. Chi-square test and student $t$ test was used for assessment of level of significance.

Table 1: $p$-value for the demographic details in between the patients of the two groups

\begin{tabular}{llccc}
\hline Parameter & & Plain & Hyperbaric & p-value \\
\hline Mean age (years) & & 61 & 59 & 0.145 \\
Mean height (cm) & & 166.5 & 164.2 & 0.125 \\
Mean weight (kg) & & 72 & 70.5 & 0.525 \\
\multirow{2}{*}{ ASA } & Type I & 9 & 15 & 0.512 \\
\multirow{2}{*}{ Speciality of surgery } & Type II & 31 & 25 & \\
& Gynae & 23 & 25 & 0.536 \\
& Urology & 17 & 15 & \\
\hline
\end{tabular}

Table 2: $\mathrm{p}$-value for block characteristics in patients in the two study groups

\begin{tabular}{lccc}
\hline Parameter & Plain & Hyperbaric & p-value \\
\hline Mean onset to T10 $(\min )$ & 10.5 & 5.2 & $0.001^{*}$ \\
Mean time for maximum block $(\min )$ & 26.2 & 26.2 & 0.300 \\
Mean duration at T10 $(\min )$ & 26.8 & 110.5 & $0.001^{*}$ \\
Mean sensory regression $(\min )$ & 272.5 & 242.5 & $0.004^{*}$ \\
Mean motor regression $(\min )$ & 180.1 & 120.2 & $0.002^{*}$ \\
\hline
\end{tabular}

*: Significant

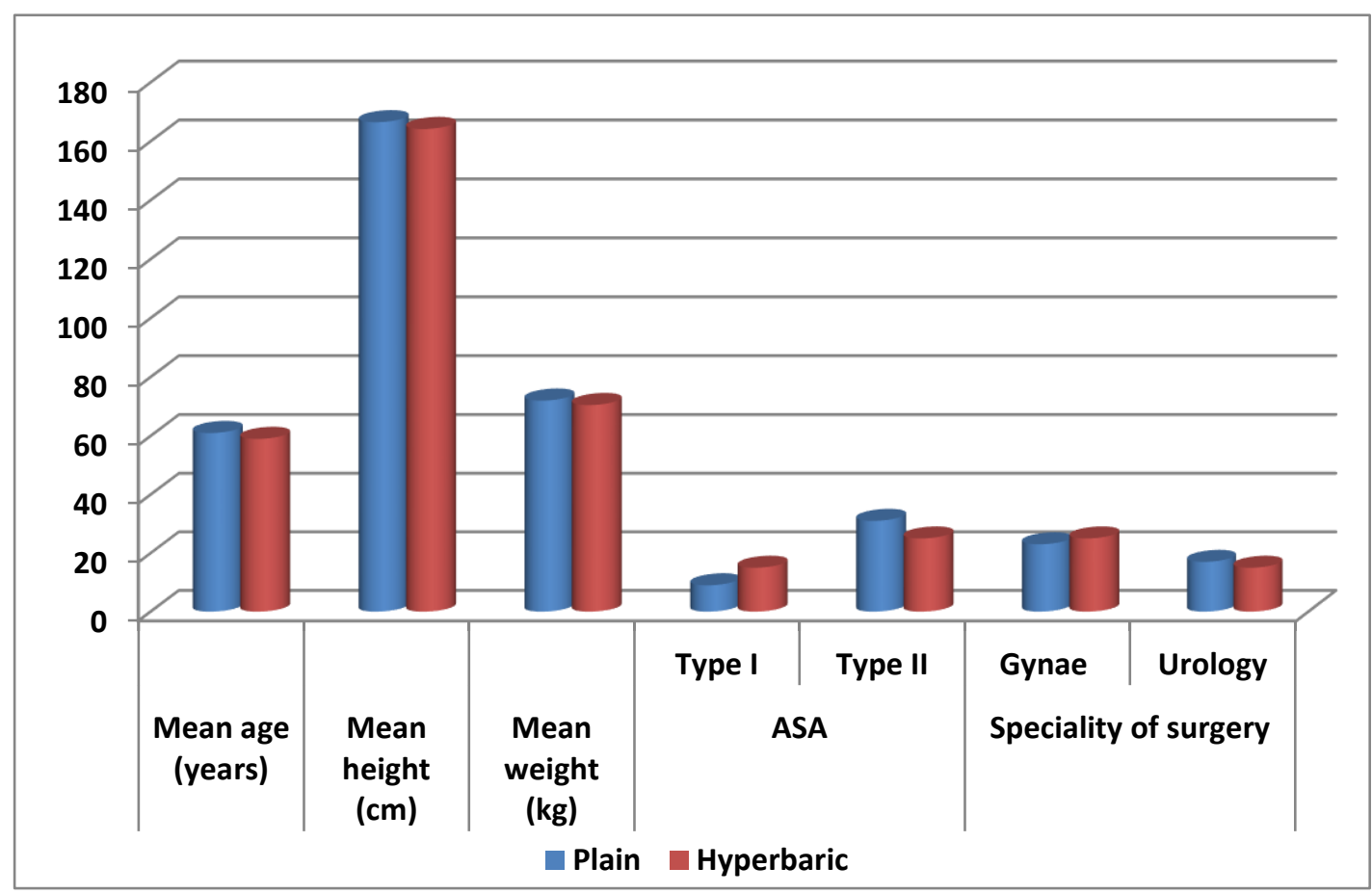

Graph 1: Demographic details of the patients of both the groups 


\section{RESULTS}

Graph 1 highlights the demographic details of the patients of both the study groups. Mean age of the patients in group receiving plain and hyperbaric anaesthetic solution was 61 and 59 years respectively. Mena height of the patients in the two study groups was 166.5 and $164.2 \mathrm{~cm}$ respectively. 72 and $70.5 \mathrm{~kg}$ was the mean weight of the patients in the two study groups. Table 1 shows the $p$-value for the demographic details of the patients. Non-significant results were obtained while comparing the demographic parameters in between the two study groups. Graph 2 shows the block characteristics in patients in the two study groups. Mean duration of onset of anaesthesia to T10 was 10.5 minutes and 5.2 minutes and patients in plain and hyperbaric group respectively. Table 2 highlights the p-value for block characteristics in patients in the two study groups. Significant differences were obtained while comparing the mean time for motor regression and mean time for sensory regression in between the two study groups.

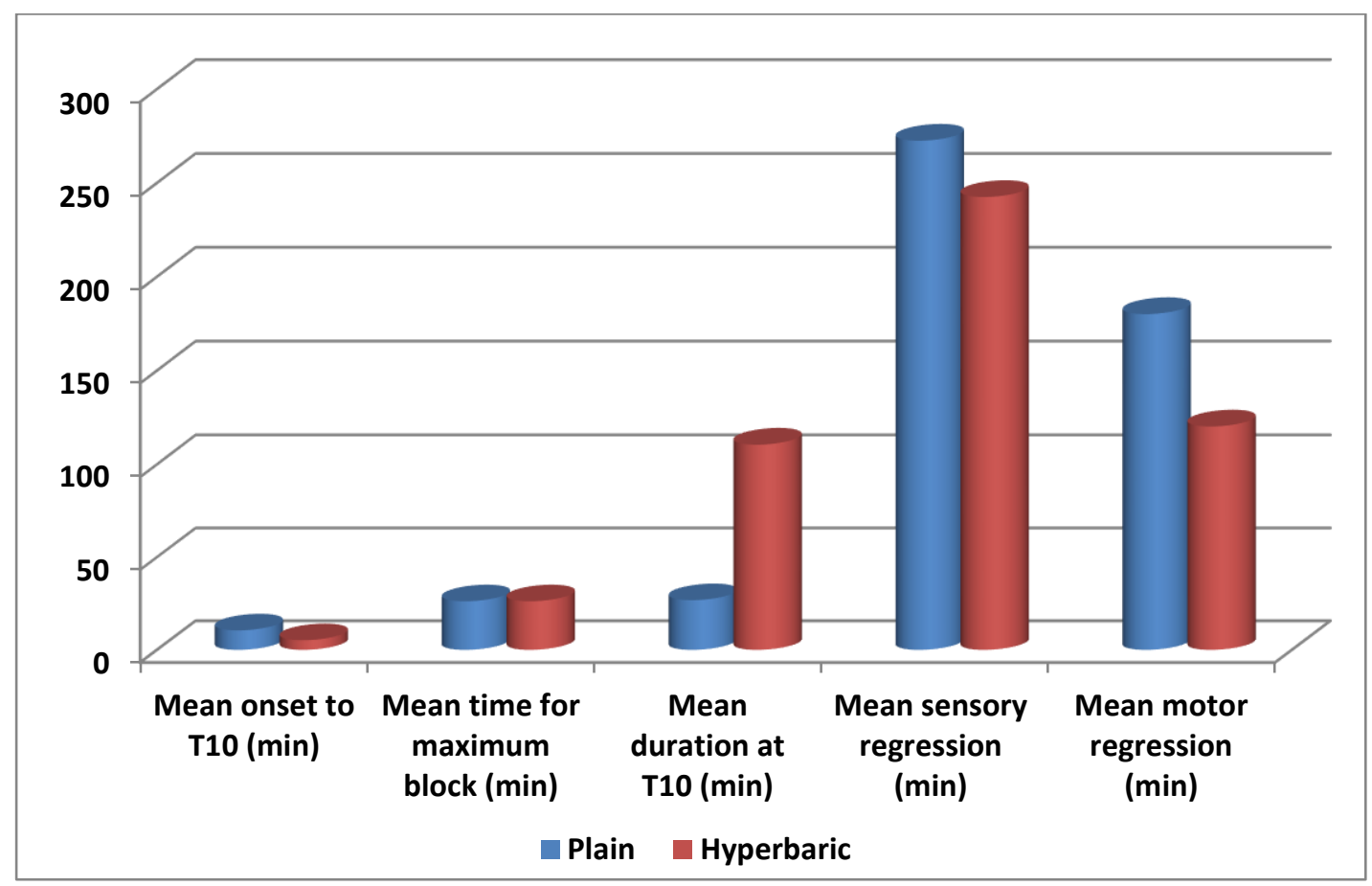

Graph 2: Block characteristics in patients in the two study groups

\section{DISCUSSION}

One of the effective, safer, cheaper means of giving anaesthesia is the spinal anaesthesia which provides rapid action and is very reliable in patients undergoing elective surgical procedures. ${ }^{5}$ Lignocaine, bupivacaine, levobupivacaine, and ropivacaine comprises the various spectrum of anaesthetic solution which have been in routines practice since past so many years in patients undergoing spinal anaesthesia. ${ }^{6,7}$ Because of its reduced side-effects on the body as whole and on specific systems of the body like the cardio system, nervous system and minimal postsurgical effects, ropivacaine is increasingly becoming popular. ${ }^{8}$ Ropivacaine has lower lipid solubility and produces varying degree of motor and sensory blockage when administered in isobaric form while when given in admixed concentration with glucose solution has a variable effect. ${ }^{9}$ Hence; we comparatively evaluated the effectiveness of plain and hyperbaric ropivacaine for providing subarachnoid block in patients undergoing elective surgeries.

In the present study, hyperbaric solution of ropivacaine was found to produce more effective and more consistent block in comparison with the plain one. A wider spread to a higher median level was observed with the addition of glucose with variation in sensory and motor block occurring to a lesser extent. Dar et al compared and evaluated the effectiveness of intrathecal hyperbaric ropivacaine with hyperbaric bupivacaine for patients undergoing limb and hip surgeries. They analyzed 200 patients of
40 to 75 years of age and randomly divided randomly them into two study groups. One group was given Ropivacaine and other was given Bupivacaine. Three-point scale was used for the assessment for onset and duration of sensory blockage. They observed that in patients of ropivacaine group, the mean onset of sensory blockage and motor block was significantly lower in comparison with the bupivacaine group. In the patients of ropivacaine group, the total mean duration of sensory block was significantly lesser in comparison with the bupivacaine group. From the result, they concluded that when used intrathecally, the effectiveness of hyperbaric bupivacaine is faster in comparison with hyperbaric ropivacaine. ${ }^{10} \mathrm{McNamee}$ et al compared the effectiveness of plain ropivacaine with plain bupivacaine in patients undergoing total hip arthroplasty with spinal anaesthesia. They analyzed 66 patients and randomly divided them into two main groups. First group comprised of patients receiving ropivacaine while the other consisted of patients receiving bupivacaine. At dermatome level T10, the onset and quantity of duration of anaesthesia was determined. They observed no statistical significant difference between the onset of motor and sensory block in between the two study groups. From the above results, they concluded that well toleration of administration of ropivacaine and bupivacaine occurs when given intrathecally in patients undergoing total hip arthroplasty. However, a more progressive and rapid sensory and motor functions occur in patients receiving ropivacaine. ${ }^{11}$ 
Kulkarni et al evaluated and compared the effectiveness of hyperbaric ropivacaine with bupivacaine for Spinal anaesthesia. They evaluated 80 patients who underwent elective infraumbilical surgeries under spinal anaesthesia. They randomly divided the patients into two study groups. First group comprised of patients who received hyperbaric ropivacaine while the other group consisted of patients who received hyperbaric bupivacaine. They observed that a slower onset of sensory block in patients receiving ropivacaine in comparison patients receiving bupivacaine. From the results, they concluded that shorter duration of spinal anaesthesia is provided by ropivacaine. ${ }^{12}$ Chung et al evaluated the effectiveness and safety of hyperbaric ropivacaine and hyperbaric bupivacaine in providing spinal anaesthesia in patients undergoing elective caesarean delivery. They analyzed 60 patients undergoing elective caesarean surgeries and divided randomly into two study groups. One group included patients receiving hyperbaric ropivacaine while the other group comprised of patients receiving hyperbaric bupivacaine. They observed that in patients receiving ropivacaine longer reaching to peak levels in terms of onset time of sensory block. From the results, they concluded that more effective spinal anaesthesia was provided by hyperbaric ropivacaine in comparison to patients who receive hyperbaric bupivacaine. ${ }^{13}$

\section{CONCLUSION}

From the above results, it can be concluded that in comparison with the hyperbaric type, plain ropivacaine has been observed to be associated with less favourable pattern of block. However, further studies are recommended.

\section{REFERENCES}

1. Van Kleef JW, Veering BT, Burm AG. Spinal anesthesia with ropivacaine: a double-blind study on the efficacy and safety of $0.5 \%$ and $0.75 \%$ solutions in patients undergoing minor lower limb surgery. Anesth Analg 1994; 78: 1125-30.

2. Wahedi W, Nolte H, Klein P. Ropivacaine for spinal anesthesia. A dose-finding study. Anaesthetist 1996; 45: 737-44.

3. Gautier PE, De Kock M, Van Steenberge A, et al. Intrathecal ropivacaine for ambulatory surgery. Anesthesiology 1999; 91: 1239-45.

4. McNamee DA, Parks L, McClelland AM, et al. Intrathecal ropivacaine for total hip arthroplasty: double-blind comparative study with isobaric $7.5 \mathrm{mg} \cdot \mathrm{ml} 1$ and $10 \mathrm{mg} \cdot \mathrm{ml} 1$ solutions. $\mathrm{Br} \mathrm{J}$ Anaesth 2001; 87: 743-7.

5. Gupta R, Verma R, Bogra J, Kohli M, Raman R, Kushwaha JK. A comparative study of intrathecal dexmedetomidine and fentanyl as adjuvants to bupivacaine. J Anaesthesiol Clin Pharmacol 2011;27:339-43.

6. Fettes PD, Hocking G, Peterson MK, Luck JF, Wildsmith JA. Comparison of plain and hyperbaric solutions of ropivacaine for spinal anaesthesia. Br J Anaesth 2005;94:107-11.

7. Kallio H, Snäll EV, Tuomas CA, Rosenberg PH. Comparison of hyperbaric and plain ropivacaine $15 \mathrm{mg}$ in spinal anaesthesia for lower limb surgery. Br J Anaesth 2004;93:664-9.

8. Yegin A, Sanli S, Hadimioglu N, Akbas M, Karsli B. Intrathecal fentanyl added to hyperbaric ropivacaine for transurethral resection of the prostate. Acta Anaesthesiol Scand2005;49:401-5. 9. Hocking G, Wildsmith JA. Intrathecal drug spread. Br J Anaesth 2004;93: 568-78.

10. Dar FA, Mushtaq MB, Khan UM. Hyperbaric spinal ropivacaine in lower limb and hip surgery: A comparison with hyperbaric bupivacaine. J Anaesthesiol Clin Pharmacol. 2015 OctDec;31(4):466-70.

11. McNamee DA, McClelland AM, Scott S, Milligan KR, Westman L, Gustafsson U. Spinal anaesthesia: comparison of plain ropivacaine $5 \mathrm{mg} \mathrm{ml}(-1)$ with bupivacaine $5 \mathrm{mg} \mathrm{ml}(-1)$ for major orthopaedic surgery. Br J Anaesth. 2002 Nov;89(5):702-6.

12. Kulkarni KR, Deshpande S, Namazi I, Singh SK, Kondilya K. A comparative evaluation of hyperbaric ropivacaine versus hyperbaric bupivacaine for elective surgery under spinal anesthesia. J Anaesthesiol Clin Pharmacol. 2014 Apr;30(2):23842.

13. Chung CJ, Choi SR, Yeo KH, Park HS, Lee SI, Chin YJ. Hyperbaric spinal ropivacaine for cesarean delivery: a comparison to hyperbaric bupivacaine. Anesth Analg. 2001 Jul;93(1):157-61.

\section{Source of Support: Nil. Conflict of Interest: None Declared.}

Copyright: (c) the author(s) and publisher. IJMRP is an official publication of Ibn Sina Academy of Medieval Medicine \& Sciences, registered in 2001 under Indian Trusts Act, 1882.

This is an open access article distributed under the terms of the Creative Commons Attribution Non-commercial License, which permits unrestricted non-commercial use, distribution, and reproduction in any medium, provided the original work is properly cited.

Cite this article as: Ajay Batra. Assessment of Plain and Hyperbaric Solutions of Ropivacaine in Patients Receiving Spinal Anaesthesia for Elective Surgeries: A Comparative Study. Int J Med Res Prof. 2016; 2(5):80-83. 